\title{
Datts, Mario: Parteikommunikation im Zeitalter von Social Media. Eine empirische Untersuchung der Facebooknutzung durch die Kreisverbände der deutschen Parteien
}

\author{
Baden-Baden: Nomos 2020. 260 Seiten. Preis: $€ 54$ (e-book)
}

\section{Paula Nitschke}

Angenommen: 9. Dezember 2020 / Online publiziert: 18. Dezember 2020

(C) Der/die Autor(en) 2020

Mario Datts untersucht in einer breit angelegten Studie die Facebook-Nutzung durch die Kreisverbände der deutschen Parteien. Der Politikwissenschaftler fragt in seiner am Graduiertenkolleg „Linkage in Democracy“ an der HHU Düsseldorf entstandenen Dissertation, in welchem Umfang die Kreisverbände der relevanten deutschen Parteien Facebook nutzen und welche Faktoren diese Nutzung beeinflussen. Den Leser erwartet eine konsequent empirisch ausgerichtete Arbeit im UV-AV-Design. Die empirische Umsetzung ist gekonnt und die Ergebnisse aufschlussreich.

Die Theoriearbeit dient zur Vorbereitung der empirischen Untersuchung und hat entsprechend das Ziel, nach Erklärungsansätzen zu suchen, die für eine empirische Prüfung geeignet sind. Um den Forschungsstand zu diesem Zweck zu durchkämmen, nutzt die Studie ein organisationssoziologisches Begriffsgerüst als „Rahmentheorie“ (S. 28), wobei der Rahmen unter anderem durch den organisationssoziologischen Neo-Institutionalismus und den Influence-of-Presumed-Media-Influence-Ansatz gefüllt wird. Die Rahmentheorie konzipiert Ziele, Instrumente und Bedingungen als Prädiktoren und Organisationsstrukturen, Funktionen und Verhalten als abhängige Organisationsvariablen, wobei für den weiteren Verlauf der Studie nur die Prädiktoren relevant sind. Nachdem der theoretische Rahmen steht, werden Erkenntnisse aus der Parteienforschung und der sozialwissenschaftlichen Internetforschung entlang der Prädiktoren aufgearbeitet. Etwas versteckt in Unterkapitel 2.3.4 wird der Forschungsstand zur Facebook-Nutzung (abhängige Variable) behandelt. Der entworfene theoretische Rahmen ist nicht vollständig rund, da die Integration der unterschiedlichen theoretischen Ansätze nicht immer reibungslos gelingt. So ist zum Beispiel der hohe Stellenwert rationaler Ziele und ihre Konzeption als Prädiktor für Organisationsverhalten nicht ganz mit der Grundannahme des Neo-Institutionalis-

Dr. P. Nitschke $(\bowtie)$

Universität Augsburg, Universitätsstraße 10, 86159 Augsburg, Deutschland

E-Mail: paula.nitschke@phil.uni-augsburg.de 
mus zu vereinbaren, wonach Strukturen und Handeln das Ergebnis von Legitimitätsstreben sind. Insgesamt folgt die Theoriearbeit aber einer klaren Struktur und erfüllt vor allem den Zweck, den sie für die Studie hat, nämlich die Strukturierung der umfangreichen empirischen Untersuchung anhand zentraler Begriffe.

Um Theorie und Empirie zu verbinden, wird in Kapitel drei ein Untersuchungsmodell mit vier Untermodellen erstellt. Das Modell operationalisiert FacebookNutzung über die abhängigen Modellvariablen Facebook-Präsenz, sowie Höhe der Informations- und Dialogaktivitäten. Als unabhängige Modellvariablen dienen die zuvor herausgearbeiteten Prädiktoren, die weiter ausdifferenziert werden. So wird ein Anreizmodell der Ziele (vote, policy, member seeking und Disintermediation) von einem Koorientierungsmodell der Ziele (andere Parteien, Einheiten der eigenen Partei und NGOs) unterschieden.

Organisationsinstrumente werden in die Kategorien Strukturen und Ressourcen unterteilt und über sieben Variablen operationalisiert. Im Teilmodell Organisationsbedingungen werden sozialräumliche Kennwerte und ihre Wahrnehmung durch die Kreisverbände sowie die Organisationskultur (Populismus: gemessen über Zugehörigkeit zur AfD) berücksichtigt. Einen Überblick über alle 32 Hypothesen im Modell zu behalten, ist für den Lesenden anspruchsvoll. Eine Hilfe ist die grafische Übersicht des Modells auf S. 100.

Gegenstand der empirischen Untersuchung waren die Facebook-Accounts der Kreisverbände aller zum Erhebungszeitraum im Bundestag vertretenen Parteien, wobei die Nutzung über einen Zeitraum von einem Jahr erfasst wurde. Da keine Listen der Kreisverbände der Parteien vorliegen, wurden in einer aufwendigen Recherche 2370 Kreisverbände als Grundgesamtheit identifiziert und eine Vollerhebung angestrebt. Die Facebook-Nutzung wurde automatisiert mittels eigens entwickelter Programme erhoben. Die Daten für die unabhängigen Variablen stammen aus einer Online-Befragung und Zensusdaten. Es wurden Regressionsanalysen gerechnet, wobei wegen der Vielzahl an Erklärungsfaktoren zunächst die Teilmodelle getestet wurden. Datenerhebung, - aufbereitung und - analyse sind sorgfältig durchgeführt, transparent dokumentiert und das entwickelte Programm (inklusive Quellcode) wird als Service für die scientific community auf der Verlagsseite zur Verfügung gestellt.

Der Ergebnisteil liefert zuerst eine deskriptive Beschreibung der Facebook-Aktivitäten der Kreisverbände, die bis ins Jahr 2008 zurück reicht und überaus aufschlussreich ist. Es zeigt sich überdeutlich, wie sehr die AfD auf der kommunalen Parteiebene Facebook dominiert: Ihre Beiträge übersteigen die Summe der Beiträge aller anderen Parteien. Der informationsfreudigste Kreisverband der AfD veröffentlichte sogar mehr Beiträge als die gesamte CSU. Bei der anschließenden Diskussion der Erklärungsfaktoren zeigt das Anreizmodell der Ziele die höchste Erklärungskraft, auch Organisationsinstrumente und Bedingungen haben Einfluss. Lediglich das Koorientierungsmodell erweist sich nicht als signifikant. Auf Ebene der 32 Indikatoren und der drei Nutzungsaspekte (Präsenz, Information, Dialog), werden viele interessante Einzelbefunde diskutiert.

Die Studie fügt dem Forschungsstand einen wichtigen Baustein hinzu, da sie eine umfangreiche Vermessung der Facebook-Nutzung eines Organisationstyps bietet, der nur selten auf der Agenda der politischen Internetforschung steht. Mit ihren zahlreichen Einzelbefunden ist sie eine hilfreiche Informationsquelle zu vielen Teil- 
aspekten rund um das Thema. Die Arbeit kann PraktikerInnen der Parteien Orientierung bieten, wobei die Botschaft klar lautet, dass sie der AfD nicht den wichtigen Kommunikationsraum Internet überlassen dürfen. Die Arbeit ist aber auch für Kommunikations- und PolitikwissenschaftlerInnen von Interesse, die empirische Studien planen oder auf Grundlage getesteter Hypothesen Theoriebildung betreiben möchten.

Funding Open Access funding enabled and organized by Projekt DEAL.

Open Access Dieser Artikel wird unter der Creative Commons Namensnennung 4.0 International Lizenz veröffentlicht, welche die Nutzung, Vervielfältigung, Bearbeitung, Verbreitung und Wiedergabe in jeglichem Medium und Format erlaubt, sofern Sie den/die ursprünglichen Autor(en) und die Quelle ordnungsgemäß nennen, einen Link zur Creative Commons Lizenz beifügen und angeben, ob Änderungen vorgenommen wurden.

Die in diesem Artikel enthaltenen Bilder und sonstiges Drittmaterial unterliegen ebenfalls der genannten Creative Commons Lizenz, sofern sich aus der Abbildungslegende nichts anderes ergibt. Sofern das betreffende Material nicht unter der genannten Creative Commons Lizenz steht und die betreffende Handlung nicht nach gesetzlichen Vorschriften erlaubt ist, ist für die oben aufgeführten Weiterverwendungen des Materials die Einwilligung des jeweiligen Rechteinhabers einzuholen.

Weitere Details zur Lizenz entnehmen Sie bitte der Lizenzinformation auf http://creativecommons.org/ licenses/by/4.0/deed.de.

Dr. Paula Nitschke ist akademische Rätin auf Zeit am Institut für Medien, Wissen und Kommunikation der Universität Augsburg. 